\section{(6) OPEN ACCESS}

\title{
Neuromuscular adjustments of the knee extensors and plantar flexors following match-play tennis in the heat
}

\author{
Julien D Périard, Olivier Girard, Sébastien Racinais
}

\section{Athlete Health and} Performance Research Centre, ASPETAR, Qatar Orthopaedic and Sports Medicine Hospital, Doha, Qatar

\section{Correspondence to} Dr Julien D Périard, AspetarQatar Orthopaedic and Sports Medicine Hospital, Research and Education Centre, Doha PO Box 29222, Qatar; julien.periard@aspetar.com

Accepted 24 December 2013

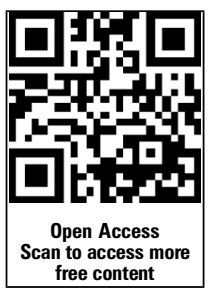

CrossMark

To cite: Périard JD, Girard O, Racinais S. Br J Sports Med 2014:48: i45-i51.
ABSTRACT

Objectives This study tested the hypothesis that impairments in lower limb maximal strength and voluntary activation (VA) are exacerbated following match-play tennis in hot compared with cool conditions. Methods Torque and VA were evaluated during brief $(5 \mathrm{~s})$ and sustained (20 s) maximal voluntary isometric contractions of the knee extensors (KE) and plantar flexors (PF) in 12 male tennis players before (pre) and after (post, $24 \mathrm{~h}$ and $48 \mathrm{~h}$ ) $\sim 115$ min of play in hot $\left(\sim 37^{\circ} \mathrm{C}\right)$ and cool $\left(\sim 22^{\circ} \mathrm{C}\right)$ conditions.

Results Rectal temperature was higher following play in hot than in cool $\left(\sim 39.2\right.$ vs $\left.\sim 38.5^{\circ} \mathrm{C} ; p<0.05\right)$. Torque production decreased from prematch to postmatch during the brief and sustained contractions in hot (KE: 22\%; PF: 13\%) and cool (KE: 9\%, PF: $\sim 7 \%)(p<0.05)$. KE strength losses in hot were greater than in cool $(p<0.05)$ and persisted for $24 h(p<0.05)$. Postmatch brief and sustained KE VA was lower in hot than in cool $(p<0.05)$, in which VA was maintained. PF VA was maintained throughout the protocol. Peak twitch torque and maximum rates of torque development and relaxation in the KE and PF were equally reduced postmatch relative to prematch in hot and cool conditions $(p<0.05)$, and were restored near baseline within $24 \mathrm{~h}$.

Conclusions Neuromuscular system integrity of the lower limbs is compromised immediately following matchplay tennis in hot and cool conditions due to the development of peripheral fatigue. The larger and persistent KE strength losses observed under heat stress are associated with greater levels of central fatigue especially during sustained contractions.

\section{INTRODUCTION}

Tennis is an intermittent activity that interposes short bouts (3-10 s) of high-intensity exercise interspersed with light activity or rest $(20-25 \mathrm{~s})$ for a protracted period of time $(1-5 \mathrm{~h}) .^{1-3}$ The cumulative effect of repeating these high-intensity efforts is manifested by the development of fatigue and suboptimal performances. ${ }^{45}$ Owing to the multifactorial nature of the game, fatigue can originate from a variety of sources (eg, cardiovascular, metabolic, neural or psychological) and/or the attainment of various states (eg, hyperthermia, dehydration or hypoglycaemia).

From a neuromuscular perspective, fatigue is defined as an exercise-induced decrement in the capacity to produce/maintain maximal force or power. ${ }^{6}$ It is purported that sources proximal (ie, central fatigue) and distal (ie, peripheral fatigue) to the neuromuscular junction modulate this decrement. Correspondingly, it has been reported that neuromuscular function deteriorates during and following prolonged $(\sim 3 \mathrm{~h})$ match-play tennis in temperate conditions. ${ }^{7-10}$ More specifically, it was shown that maximal strength of the knee extensors (KE) and plantar flexors (PF) decreases during brief maximal voluntary isometric contractions (MVCs) in association with a reduction in voluntary muscle activation (VA), adjustments in contractile function and/or the development of low-frequency fatigue, depending on the muscle group. ${ }^{7-10}$ This latter form of fatigue is characterised by a proportionately greater loss of force at low frequencies of muscle stimulation and a slow recovery over the course of several hours or even days. ${ }^{11}$ Alternatively, reductions in VA measured via twitch interpolation are indicative of a loss in neural drive to active muscles during an effort. Accordingly, these central and peripheral alterations have the potential to negatively influence the efficiency of on-court movements and stroke proficiency, ${ }^{9}$ especially during multiple-match tournament play.

Interestingly, the recovery kinetics of the KE and PF following match-play tennis remain relatively unknown, having only been studied $30 \mathrm{~min}$ after play. ${ }^{8-10}$ Although a recent study has shown that bilateral leg press force is reduced immediately after the first match of a 3-day tournament and remains depressed prior to the start of play in the following matches (ie, days 2 and 3), ${ }^{12}$ measures of central and peripheral fatigue were not conducted. As such, the origin of fatigue, along with the extent and timeframe of these potential alterations, as well as the contribution of different muscle groups were not elucidated.

Moreover, there is strong evidence to suggest that central fatigue is exacerbated by hyperthermia per se (ie, passive heating) ${ }^{13-18}$ However, the precise influence of exercise-induced hyperthermia on central activation deficit remains contentious. For example, the ability to generate force during a sustained MVC was shown to decline after constant rate exercise in hot relative to cool conditions, due to a hyperthermia-induced reduction in VA. ${ }^{19}$ Conversely, force production was similarly impaired following self-paced and incremental exercise in hot and cool conditions, with a level of central fatigue that was equivalent. ${ }^{20}{ }^{21}$ After a soccer match, a high-intensity intermittent activity more closely comparable to tennis, decrements in torque production and VA were similar between matches played in hot and cool conditions, and were restored within $24 \mathrm{~h}^{22}$

Therefore, the aim of this study was to test the hypothesis that impairments in lower limb strength 
and VA are exacerbated following play in hot conditions compared with cool conditions. A secondary aim was to test hypothesis that the time course of recovery in neuromuscular function is impaired over a $48 \mathrm{~h}$ period. From a tennis performance perspective, acute (ie, immediately postmatch) and delayed (ie, 24$48 \mathrm{~h}$ into recovery) lower-limb strength losses, along with central activation deficits following match-play in the heat, could have important implications for competition preparation and recovery.

\section{METHODS}

\section{Subjects}

Twelve male players with an International Tennis Federation number of 1 to 3 (ie, nationally ranked players, or having professional tournament experience) participated in the study. Mean age, height and body mass were $22 \pm 4$ years, $183.5 \pm 7.7 \mathrm{~cm}$ and $80.8 \pm 9.5 \mathrm{~kg}$. The participants were informed of the study aims, requirements and risks before providing their written informed consent.

\section{Study design}

The participants played two counter-balanced simulated tennis matches on hard-court surfaces separated by 72 or $144 \mathrm{~h}$. They were paired according to their level of play and competed against the same opponent in each match. One match was played indoors in temperate conditions (cool: $21.8 \pm 0.1^{\circ} \mathrm{C}, 72.3$ $\pm 3.2 \%$ relative humidity) and the other outside in hot conditions (hot: $36.8 \pm 1.5^{\circ} \mathrm{C}, 36.1 \pm 11.3 \%$ relative humidity). The matches consisted of $20 \mathrm{~min}(2 \times 10 \mathrm{~min})$ of effective play: the percentage of time spent within play and excluding the time between points and games. One to 3 days prior to the start of the study the participants visited the testing and playing venue where they were thoroughly familiarised with the testing procedures and neuromuscular function assessment protocol (see Motor nerve stimulation section) until they felt accustomed with the equipment (ie, coefficient of variation in three successive MVC trials lower than 5\%).

\section{Experimental protocol}

Upon arrival on match days (09:00), participants voided and inserted a telemetric temperature pill (VitalSense, Mini Mitter, Respironics, Herrsching, Germany) in the rectum (the length of a gloved index finger beyond the anal sphincter) for the measurement of core temperature. A standardised warm-up of running at $9 \mathrm{~km} / \mathrm{h}$ for $5 \mathrm{~min}$ on the indoor court was then performed. This was followed by the pre-exercise (pre) neuromuscular function assessment (see Neuromuscular function section). This assessment, along with the 24 and $48 \mathrm{~h}$ postmatch assessments, was preceded by a standardised warm-up consisting of 10 isometric contractions of $4 \mathrm{~s}$ in duration interspaced with $10-20$ s of recovery. Contraction intensity was progressively selfadjusted by the participant to attain maximal torque in the last three contractions. The neuromuscular function assessment was repeated $\sim 20$ min after play (post). Following the neuromuscular assessment, the participants performed a $10 \mathrm{~min}$ tennisspecific warm-up consisting of various exchanges and serves in the condition of play. Core temperature and body mass were recorded prematch and postmatch. During play, the participants consumed water and a commercially available sport drink (Gatorade, Chicago, Illinois, USA) ad libitum. On the days when the participants did not play they performed the running warm-up and the neuromuscular assessment (24 and $48 \mathrm{~h}$ ) at the same time of day as prematch, and then followed a standardised training programme led by a tennis coach ( 60 min of drills and exchanges).

\section{Neuromuscular function}

The neuromuscular assessment protocol is presented in figure 1 . The protocol was performed in a counter-balanced order between participants, once to evaluate the KE and once to evaluate the $\mathrm{PF}$ of the right leg. First, a brief $(5 \mathrm{~s})$ MVC was performed on which a paired stimulus (doublet, $100 \mathrm{~Hz}$ ) was superimposed. This was followed by a doublet and three single pulses on relaxed muscle, each interspersed by $5 \mathrm{~s}$. After repeating this initial part of the protocol and following a $60 \mathrm{~s}$ rest interval, a sustained $(20 \mathrm{~s}) \mathrm{MVC}$ was performed with a doublet superimposed at $\sim 2$ and $\sim 18 \mathrm{~s}$. In addition, a potentiated doublet was delivered $5 \mathrm{~s}$ after the sustained MVC. The sustained MVC was included in the assessment as it is suggested to better evaluate central activation failure. ${ }^{23}$ The duration of the entire neuromuscular assessment (ie, for both muscle groups) was $\sim 10 \mathrm{~min}$ and was conducted in a temperate environment $\left(\sim 22^{\circ} \mathrm{C}\right)$. During all MVCs, the participants were instructed to reach maximal torque as quickly as possible and maintain this level for the duration of the contraction. They were strongly encouraged with verbal feedback and a visual display of the torque production.

\section{Torque measurements}

For KE torque measurement, participants were seated upright on a custom-built adjustable chair with the hips and knees flexed at $90^{\circ}$. Restraining straps placed across the chest and hips secured the participants in the chair to prevent extraneous movement, while the dynamometer (Captels, St Mathieu de Treviers, France) was attached $3-5 \mathrm{~cm}$ above the tip of the lateral malleoli. PF torque was measured using a dynamometric pedal (Captels, St Mathieu de Treviers, France) with the participants seated upright with the hips, knee and ankle flexed at $90^{\circ}, 100^{\circ}$ and $90^{\circ}$, respectively. The foot of the leg performing the MVC was secured to the dynamometric pedal with two restraining straps. During all contractions, the torque signals were amplified, sent through an A/D board and sampled at $2000 \mathrm{~Hz}$ by commercially available hardware and software (MP35 and BSL Pro V.3.6.7, Biopac Systems Inc., Santa Barbara, California, USA).

\section{Motor nerve stimulation}

A high-voltage stimulator (Digitimer DS7AH, Digitimer, Hertfordshire, England) was used to deliver a square-wave stimulus of $0.2 \mathrm{~ms}$ duration with a maximal voltage of $400 \mathrm{~V}$. The femoral nerve was stimulated by placing a cathode $(5 \mathrm{~mm}$ diameter $)$ in the inguinal crease and an anode $(5 \times 10 \mathrm{~cm}$; Medicompex SA, Ecublens, Switzerland) in the gluteal fold. The

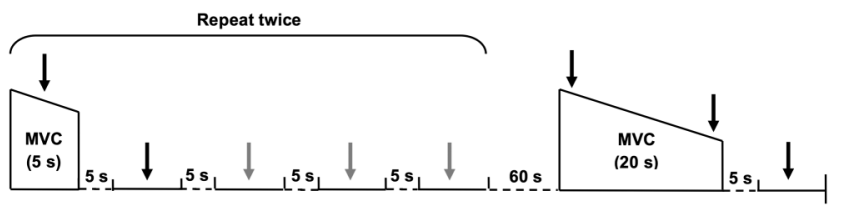

Figure 1 Neuromuscular function assessment protocol performed prior to and following $20 \mathrm{~min}$ of effective match-play tennis $(2 \times 10 \mathrm{~min})$ in cool and hot conditions with the knee extensors and plantar flexors. A $5 \mathrm{~s}$ maximal voluntary isometric contraction (MVC) was performed and superimposed with motor nerve stimulation. This was followed by four stimulations at rest. After repeating this procedure, a $20 \mathrm{~s} \mathrm{MVC}$ was performed with stimulations superimposed at 2 and $18 \mathrm{~s}$. A final potentiated stimulation was evoked $5 \mathrm{~s}$ after the sustained MVC. Single (1 Hz: $\downarrow)$ and paired $(100 \mathrm{~Hz}: \downarrow)$ stimulations. 
tibial nerve was stimulated using a cathode (9 $\mathrm{mm}$ diameter) placed in the popliteal cavity with compression supplied by a strap, and an anode $(5 \times 10 \mathrm{~cm})$ positioned beneath the patella. During the familiarisation session, an isometric recruitment curve using motor nerve stimulation was drawn on relaxed $\mathrm{KE}$ and PF muscles to individualise the optimal stimulus intensities. Briefly, the current was progressively increased in $10 \mathrm{~mA}$ increments until plateau occurred in maximal twitch amplitude. Supramaximal stimulations were ensured by increasing the final intensity by $50 \%$, and kept constant for each participant throughout all experimental trials.

\section{Neuromuscular data analysis}

All analyses were performed using Spike 2 Software (Cambridge Electronic Design, Cambridge, UK). During the MVCs, torque production was recorded during a $500 \mathrm{~ms}$ plateau prior to delivering the motor nerve stimulation. VA was calculated with the interpolated twitch technique using the potentiated doublet, as VA (\%) $=(1-($ superimposed doublet/resting potentiated doublet $)) \times 100 .^{24}$ Contractile characteristics were assessed from the electrically evoked resting twitch as peak twitch torque, contraction time to peak torque, half-relaxation time and maximum rates of torque development and relaxation (ie, the steepest rates of torque production and decline, respectively). The better of the two brief MVCs was selected to assess brief maximal voluntary torque production and VA, whereas the mean of the six twitches was used to analyse contractile properties.

\section{Statistical analysis}

All statistical calculations were performed using PASW software V.21.0 (SPSS, Chicago, Illinois, USA). Repeated-measures analysis of variance were performed to test the significance between and within treatments. Outcome variables were tested using Mauchly's procedure for sphericity. Whenever the data violated the assumption of sphericity, $\mathrm{p}$ values and adjusted degrees of freedom based on Greenhouse-Geisser correction were reported instead. Where significant interaction effects were established, pairwise differences were identified using the Bonferroni post hoc analysis procedure adjusted for multiple comparisons. The significance level was set at $\mathrm{p}<0.05$. All values are expressed as means \pm SD.

\section{RESULTS}

\section{Match-play responses}

Match duration, the time to complete 20 min of effective play, was longer in the hot condition $(124.2 \pm 9 \mathrm{~min})$ than in the cool condition $(107.1 \pm 19 \mathrm{~min})(\mathrm{p}<0.05)$. The increase in core temperature from prematch to postmatch was greater in the hot condition $\left(37.6 \pm 0.3\right.$ to $\left.39.4 \pm 0.5^{\circ} \mathrm{C}\right)$ relative to the cool condition $\left(37.5 \pm 0.3\right.$ to $\left.38.7 \pm 0.2^{\circ} \mathrm{C} ; \mathrm{p}<0.05\right)$. Core temperature during the neuromuscular assessment was also higher following play in the heat $\left(39.2 \pm 0.5\right.$ vs $\left.38.5 \pm 0.3^{\circ} \mathrm{C} ; \mathrm{p}<0.05\right)$. The level of body mass deficit was similar between the hot $(-0.5 \pm 1.3 \mathrm{~kg})$ and cool $(-0.3 \pm 0.3 \mathrm{~kg})$ conditions, as the $0.7 \pm 0.4 \mathrm{~L} / \mathrm{h}$ greater sweat rate observed in the hot condition was compensated for by a greater fluid consumption of $0.9 \pm 0.5 \mathrm{~L} / \mathrm{h}(\mathrm{p}<0.001)$.

\section{Maximal voluntary contractions}

Knee extension

Compared with prematch values, larger reductions in brief $\mathrm{KE}$ torque production occurred postmatch in the hot condition $(-22.0 \pm 10.9 \%)$ than in the cool condition $(-9.5 \pm 6.9 \%$; figure $2 ; \mathrm{p}<0.05)$. KE torque was also lower in the hot condition at $24 \mathrm{~h}$ relative to prematch $(-10.6 \pm 7.3 \%)$ and cool $(-11.2 \pm 9.1 \%)$ condition values $(\mathrm{p}<0.05)$. VA during the brief KE MVC was maintained throughout the cool condition, whereas it was reduced postmatch in the hot condition compared with prematch $(-11.4 \pm 9.1 \%)$ and cool $(-12.9 \pm 9.1 \%)$ condition values $(\mathrm{p}<0.05)$. Overall, KE torque production and VA were lower throughout the hot vs cool condition $(p<0.05)$.

Sustained KE torque production decreased similarly $(-5 \%$ to $12 \%$ ) throughout all MVCs (ie, from 2 to $18 \mathrm{~s}$ ) (figure 3; $\mathrm{p}<0.01$ ). As with the brief MVCs, a decrease in mean sustained torque was observed postmatch relative to prematch in hot $(-21.5 \pm 11.0 \%)$ and cool $(-8.0 \pm 7.9 \%)$ conditions $(\mathrm{p}<0.05)$. Mean KE-sustained torque production was also reduced $24 \mathrm{~h}$ after match completion in the hot condition, relative to prematch $(p<0.01)$. Torque production postmatch and at $24 \mathrm{~h}$ in
Figure 2 Torque production and voluntary activation during a brief $(5 s)$ maximal voluntary isometric contraction of the knee extensors (left panels) and plantar flexors (right panels) performed prior to (pre) and following (post, 24 and 48 h) 20 min of effective match-play tennis in cool and hot conditions. * Significantly different from pre, $p<0.05$.

tSignificantly different from cool, $\mathrm{p}<0.05$.
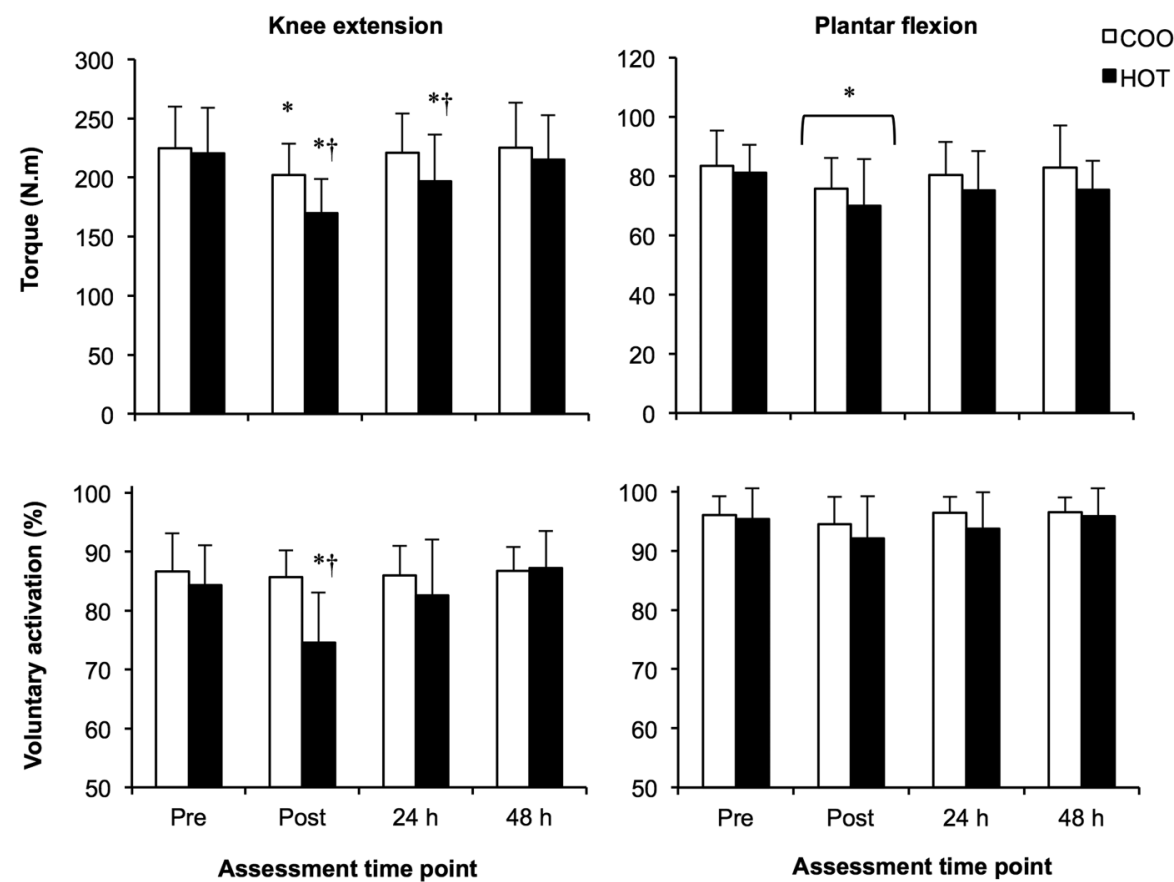

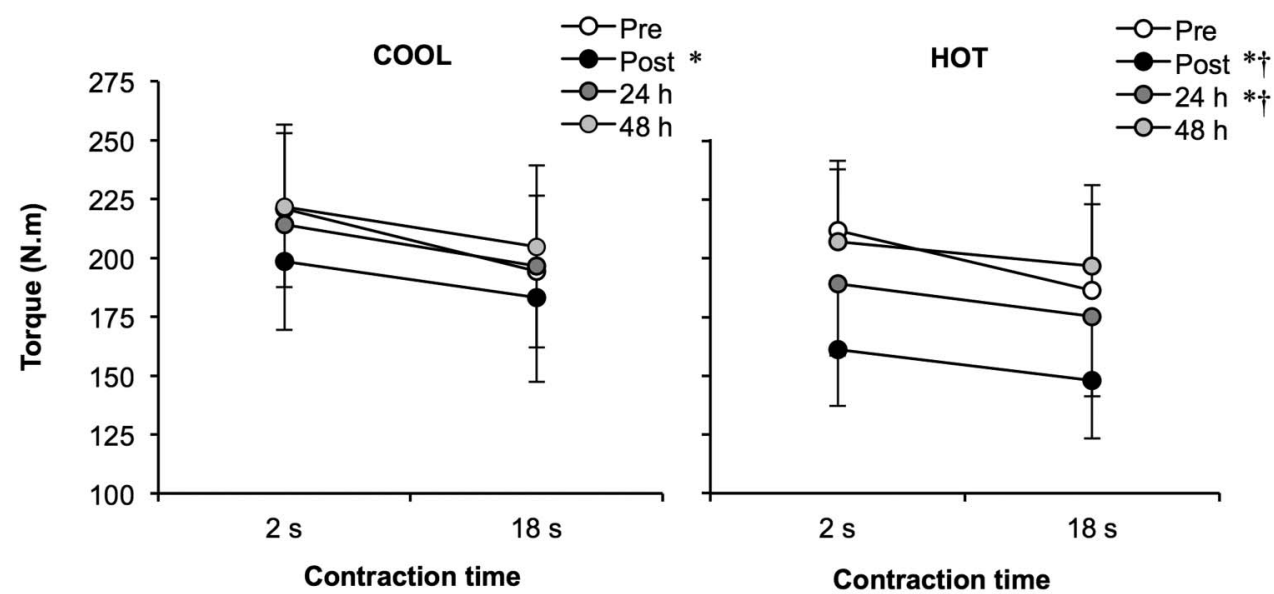

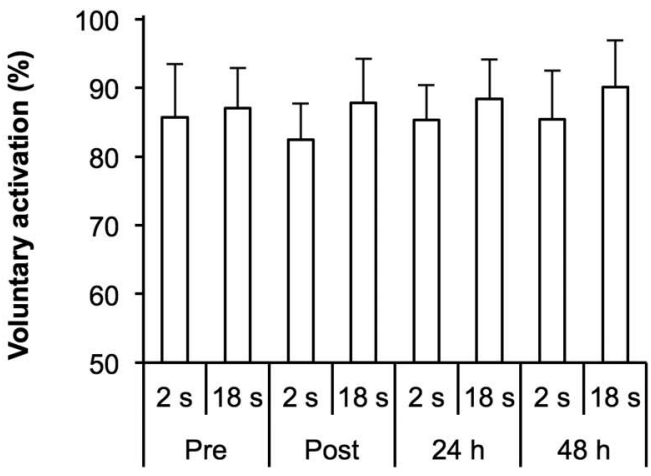

Assessment time point

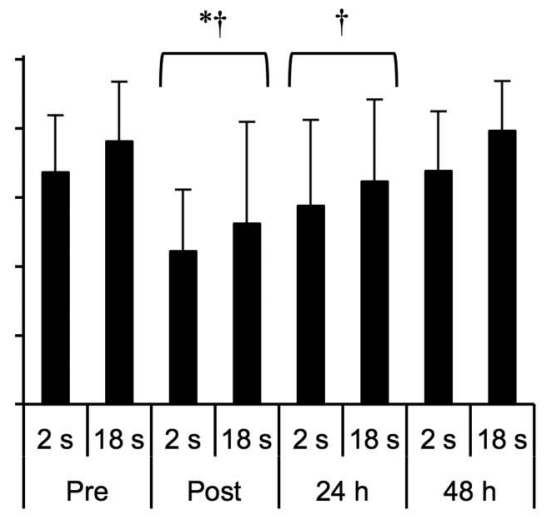

Assessment time point

Figure 3 Knee extensor torque production and voluntary activation during a sustained (20 s) maximal voluntary isometric contraction (MVC) in cool (left panels) and hot (right panels) conditions with stimulations superimposed at 2 and $18 \mathrm{~s}$. The MVCs were performed prior to (pre) and following (post, 24 and 48 h) 20 min of effective match-play tennis in each condition *Significantly different from pre, $p<0.05$. + Significantly different from cool, $\mathrm{p}<0.05$.

the hot condition was lower than in the cool condition at the same time-points $(\mathrm{p}<0.01)$. VA during the sustained MVC was lower postmatch in the hot condition compared with prematch $(-13.8 \pm 9.5 \%)$ and cool $(-12.9 \pm 12.4 \%)$ condition values $(\mathrm{p}<0.05)$. KE VA was also lower $24 \mathrm{~h}$ after play in the hot condition compared with the cool condition $(p<0.05)$. Sustained KE torque and VA were also lower throughout the hot vs cool condition $(\mathrm{p}<0.05)$.

\section{Plantar flexion}

PF torque production during the brief MVCs decreased (-11.2 $\pm 13.6 \%$ ) postmatch relative to prematch, with no difference between conditions (figure $2 ; \mathrm{p}<0.05$ ). Brief PF torque production was higher throughout the cool vs cool condition $(\mathrm{p}<0.05)$. On the other hand, VA was maintained at a similar level throughout the protocol.

Sustained PF torque production decreased by $-9 \%$ to $15 \%$ throughout all MVCs (ie, from 2 to $18 \mathrm{~s}$ ) (figure 4; $\mathrm{p}<0.01$ ). An overall reduction in mean torque was observed postmatch relative to prematch in the hot $(-14.4 \pm 19.2 \%)$ and cool $(-7.0$ $\pm 11.4 \%)$ conditions $(\mathrm{p}<0.05)$. Mean PF torque production was higher throughout the cool vs hot condition $(p<0.05)$. Although VA was slightly lower postmatch compared with prematch $(p=0.07)$ and throughout the hot condition relative to the cool condition $(p=0.06)$, statistical significance was not attained. A significant difference in VA was observed between the 2 and $18 \mathrm{~s}$ stimulation intervals, whereby the latter was lower $(\mathrm{p}<0.01)$.

\section{Contractile properties}

Peak twitch torque of the KE decreased significantly postmatch in both conditions compared with prematch values (table 1; $\mathrm{p}<0.05)$. Peak twitch torque and contraction time were higher throughout the hot vs cool condition $(\mathrm{p}<0.05)$. The maximum rates of torque development and relaxation were similarly reduced postmatch in the hot and cool conditions, relative to prematch $(\mathrm{p}<0.05)$.

Mean PF peak twitch torque decreased significantly from prematch to postmatch (table $2 ; \mathrm{p}<0.05$ ). Contraction time was lower throughout the cool vs hot condition $(\mathrm{p}<0.05)$. Mean PF maximum rates of torque development and relaxation were significantly reduced postmatch relative to prematch $(\mathrm{p}<0.05)$.

\section{DISCUSSION}

In agreement with our first hypothesis, impairments in lower limb strength and VA were exacerbated following match-play tennis in the hot condition compared with cool condition. More specifically, our results indicate that brief and sustained maximal torque production of the $\mathrm{KE}$ and $\mathrm{PF}$ was reduced immediately after play in hot and cool conditions. However, the reduction in torque was exacerbated under heat stress in the $\mathrm{KE}$ only, remaining depressed $24 \mathrm{~h}$ after match completion. The 


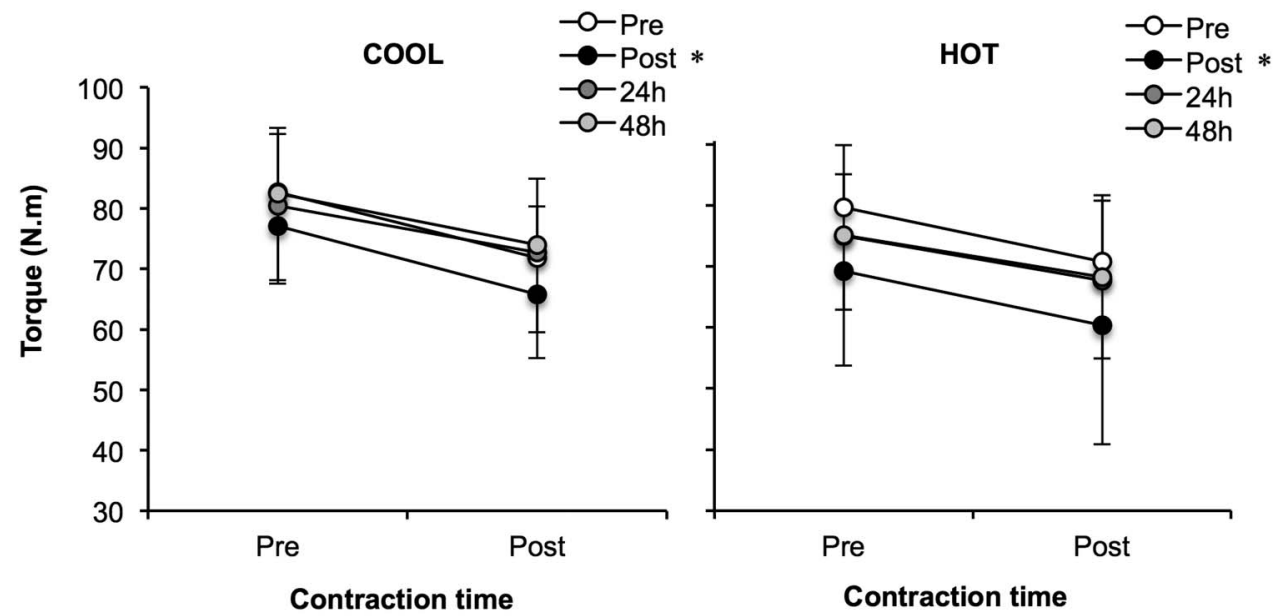

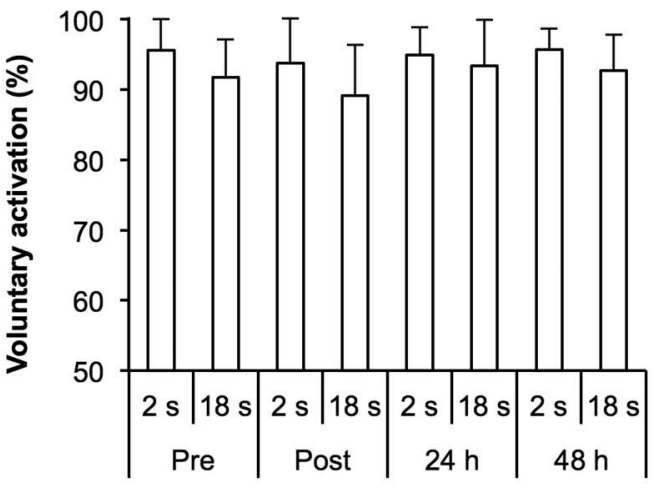

Assessment time point

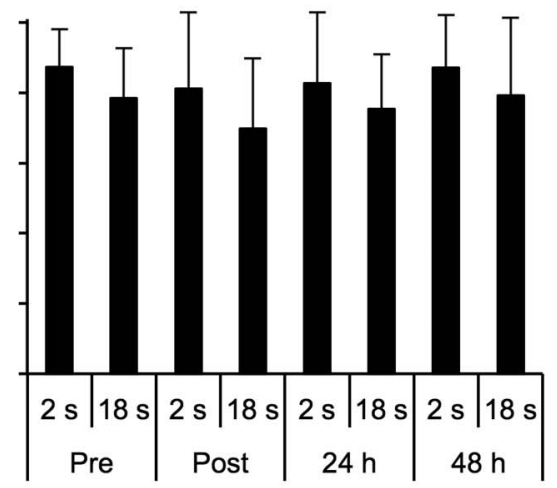

Assessment time point

Figure 4 Plantar flexor torque production and voluntary activation during a sustained (20 s) maximal voluntary isometric contraction (MVC) in cool (left panels) and hot (right panels) conditions with stimulations superimposed at 2 and $18 \mathrm{~s}$. The MVCs were performed prior to (pre) and following (post, 24 and 48 h) 20 min of effective match-play tennis in each condition. *Significantly different from pre, $p<0.05$.

loss of strength appears to have originated from a combination of central and peripheral fatigue factors in the $\mathrm{KE}$ and mainly from peripheral adjustments in the PF.

\section{Acute postmatch responses}

The KE strength losses noted in the brief $(\sim 10 \%)$ and sustained $(\sim 8 \%)$ MVCs performed after play in the cool condition are similar $(\sim 9 \%)$ to those reported during brief contractions performed after $3 \mathrm{~h}$ of match-play in similar environmental conditions. ${ }^{9} 10$ They are also similar to isometric bilateral leg press force losses $(\sim 8 \%)$ shown immediately following the first $2 \mathrm{~h}$ match of a 3-day tournament in temperate conditions. ${ }^{12}$ In the current study, the loss of KE strength postmatch was associated with a reduction in VA (figures 2 and 3 ) and adjustments in contractile function, as manifested by a reduction in peak twitch torque and slower rates of twitch torque development/relaxation (table 1). Hence, our data indicate that the adjustments in muscle contractility associated with peripheral fatigue are characterised

Table 1 Knee extensor peak twitch torque, contraction time, half-relaxation time, maximum rate of torque development and maximum rate of torque relaxation prior to (pre) and following (post, 24 and 48 h) 20 min of effective match-play tennis in cool and hot conditions

\begin{tabular}{|c|c|c|c|c|c|}
\hline \multirow{2}{*}{$\begin{array}{l}\text { Contractile } \\
\text { characteristic }\end{array}$} & \multirow[b]{2}{*}{ Condition } & \multicolumn{4}{|c|}{ Assessment time point } \\
\hline & & Pre & Post & $24 \mathrm{~h}$ & $48 \mathrm{~h}$ \\
\hline Peak twitch torque $(\mathrm{N} \mathrm{m})$ & $\begin{array}{l}\text { Cool } \\
\text { Hot }\end{array}$ & $\begin{array}{l}58.6 \pm 7.9 \\
62.4 \pm 7.3\end{array}$ & $\begin{array}{l}50.2 \pm 10.3^{*} \\
56.9 \pm 8.5^{*}\end{array}$ & $\begin{array}{l}58.2 \pm 6.3 \\
60.1 \pm 10.3\end{array}$ & $\begin{array}{l}61.5 \pm 9.9 \\
62.2 \pm 11.6\end{array}$ \\
\hline Contraction time (ms) & $\begin{array}{l}\text { Cool } \\
\text { Hot }\end{array}$ & $\begin{array}{l}74.1 \pm 8.7 \\
75.6 \pm 8.0\end{array}$ & $\begin{array}{l}69.3 \pm 9.6 \\
74.3 \pm 5.9\end{array}$ & $\begin{array}{l}75.2 \pm 7.2 \\
77.5 \pm 7.3\end{array}$ & $\begin{array}{l}75.2 \pm 6.9 \\
82.8 \pm 9.1\end{array}$ \\
\hline Half-relaxation time (ms) & $\begin{array}{l}\text { Cool } \\
\text { Hot }\end{array}$ & $\begin{array}{l}67.8 \pm 9.8 \\
70.4 \pm 10.0\end{array}$ & $\begin{array}{l}69.3 \pm 10.0 \\
73.0 \pm 9.8\end{array}$ & $\begin{array}{l}73.9 \pm 8.5 \\
77.1 \pm 14.8\end{array}$ & $\begin{array}{l}74.3 \pm 8.1 \\
76.4 \pm 12.9\end{array}$ \\
\hline Maximum rate of torque development $\left(\mathrm{N} \mathrm{m} / \mathrm{s}^{1}\right)$ & $\begin{array}{l}\text { Cool } \\
\text { Hot }\end{array}$ & $\begin{array}{l}1634 \pm 363 \\
1658 \pm 271\end{array}$ & $\begin{array}{l}1358 \pm 318^{*} \\
1438 \pm 210^{*}\end{array}$ & $\begin{array}{l}1582 \pm 204 \\
1599 \pm 223\end{array}$ & $\begin{array}{l}1663 \pm 310 \\
1551 \pm 302\end{array}$ \\
\hline Maximum rate of torque relaxation $(\mathrm{N} \mathrm{m} / \mathrm{s})$ & $\begin{array}{l}\text { Cool } \\
\text { Hot }\end{array}$ & $\begin{array}{l}-719 \pm 161 \\
-747 \pm 151\end{array}$ & $\begin{array}{l}-567 \pm 148^{*} \\
-607 \pm 132^{*}\end{array}$ & $\begin{array}{l}-671 \pm 120 \\
-704 \pm 143\end{array}$ & $\begin{array}{l}-685 \pm 149 \\
-692 \pm 191\end{array}$ \\
\hline
\end{tabular}


Table 2 Plantar flexor peak twitch torque, contraction time, half-relaxation time, maximum rate of torque development and maximum rate of torque relaxation prior to (pre) and following (post, 24 and 48 h) 20 min of effective match-play tennis in cool and hot conditions

\begin{tabular}{|c|c|c|c|c|c|}
\hline \multirow{2}{*}{$\begin{array}{l}\text { Contractile } \\
\text { Characteristic }\end{array}$} & \multirow[b]{2}{*}{ Condition } & \multicolumn{4}{|c|}{ Assessment time point } \\
\hline & & Pre & Post & $24 \mathrm{~h}$ & $48 \mathrm{~h}$ \\
\hline Peak twitch torque ( $\mathrm{N} \mathrm{m}$ ) & $\begin{array}{l}\text { Cool } \\
\text { Hot }\end{array}$ & $\begin{array}{l}16.0 \pm 3.2 \\
15.3 \pm 4.3\end{array}$ & $\begin{array}{l}14.1 \pm 3.1^{*} \\
13.8 \pm 4.2^{*}\end{array}$ & $\begin{array}{l}15.8 \pm 3.8 \\
14.7 \pm 3.0\end{array}$ & $\begin{array}{l}15.5 \pm 3.6 \\
15.6 \pm 2.9\end{array}$ \\
\hline Contraction time (ms) & $\begin{array}{l}\text { Cool } \\
\text { Hot }\end{array}$ & $\begin{array}{l}80.7 \pm 6.7 \\
80.3 \pm 9.3\end{array}$ & $\begin{array}{l}77.9 \pm 9.8 \\
83.3 \pm 8.0\end{array}$ & $\begin{array}{l}80.8 \pm 6.2 \\
83.3 \pm 8.8\end{array}$ & $\begin{array}{l}77.0 \pm 4.3 \\
80.5 \pm 8.1\end{array}$ \\
\hline Half-relaxation time (ms) & $\begin{array}{l}\text { Cool } \\
\text { Hot }\end{array}$ & $\begin{array}{l}65.8 \pm 5.9 \\
66.3 \pm 7.5\end{array}$ & $\begin{array}{l}67.4 \pm 7.8 \\
63.8 \pm 9.0\end{array}$ & $\begin{array}{l}67.9 \pm 9.7 \\
68.9 \pm 11.8\end{array}$ & $\begin{array}{l}64.1 \pm 13.1 \\
65.7 \pm 8.4\end{array}$ \\
\hline Maximum rate of torque development $(\mathrm{N} \mathrm{m} / \mathrm{s})$ & $\begin{array}{l}\text { Cool } \\
\text { Hot }\end{array}$ & $\begin{array}{l}540 \pm 158 \\
536 \pm 178\end{array}$ & $\begin{array}{l}471 \pm 107^{*} \\
473 \pm 172^{*}\end{array}$ & $\begin{array}{l}530 \pm 141 \\
495 \pm 120\end{array}$ & $\begin{array}{l}538 \pm 143 \\
513 \pm 107\end{array}$ \\
\hline Maximum rate of torque relaxation $(\mathrm{N} \mathrm{m} / \mathrm{s})$ & $\begin{array}{l}\text { Cool } \\
\text { Hot }\end{array}$ & $\begin{array}{l}-207 \pm 48 \\
-193 \pm 59\end{array}$ & $\begin{array}{l}-173 \pm 33^{*} \\
-162 \pm 51^{*}\end{array}$ & $\begin{array}{l}-192 \pm 51 \\
-180 \pm 48\end{array}$ & $\begin{array}{l}-196 \pm 51 \\
-189 \pm 33\end{array}$ \\
\hline
\end{tabular}

not only by decrements in peak twitch torque, but also by slowing of the muscle contractile speed. Interestingly, VA levels and contractile properties (peak twitch torque) in previous tennis studies were shown not to significantly differ in the KE from prematch to postmatch. ${ }^{10}$ However, the torque ratio of stimulations evoked at 20 and $80 \mathrm{~Hz}$ declined after play, as did the MVC-to- $80 \mathrm{~Hz}$ stimulation ratio. ${ }^{10}$ This led to the suggestion that peripheral and central adjustments occurred during fatigue, ${ }^{10}$ the former being associated with low-frequency fatigue, which is a form of fatigue that stems from exercises that cause muscle damage and impairments in excitation-contraction coupling. ${ }^{112526}$

In a previous study, impairments in PF brief torque production $(\sim 15 \%)$ following match-play were similarly attributed to a reduction in voluntary drive, while a decrease in peak twitch torque further confirmed the presence of skeletal muscle fatigue. ${ }^{8}$ Our results support part of these observations, in that the reduction in brief $(\sim 11 \%)$ and sustained $(\sim 7 \%)$ MVC torque in the PF was related to decrements in contractile function (table 2); however, central activation failure was not observed. Recently, Fabre et $\mathrm{al}^{7}$ reported a similar decrease ( $\sim 9 \%)$ in brief MVC force after indoor match-play tennis, along with a reduction in peak twitch force and twitch contraction time. As with the current study, they observed that VA was unaffected, even after 45 min of effective play, concluding that the loss of strength occurred concomitantly with changes in muscle contractility. Our data thus confirm these tennis-specific observations in that $\mathrm{KE}$ and $\mathrm{PF}$ torque production is compromised following play in cool conditions, yet extends them to indicate that the decrement occurs to the same extent during brief and sustained MVCs. Furthermore, the reduction in KE torque appears related to central and peripheral alterations, whereas PF strength is primarily influenced by adjustments in contractile function.

\section{Influence of heat stress}

In the hot condition, the decrease in brief $(\sim 22 \%)$ and sustained $(\sim 22 \%)$ KE torque postmatch was greater than in the cool condition (figures 2 and 3). As with the cool condition, the decrement was associated with a reduction in VA and twitch contractility (table 1). However, during both contraction lengths, the level of central activation failure was greater in the hot condition. Interestingly, while several studies have shown that passive hyperthermia is associated with reductions in VA, ${ }^{13} 14^{16-18}$ the role of exercise-induced hyperthermia in exacerbating central fatigue is unclear. Nybo and Nielsen ${ }^{19}$ initially showed that force production and VA progressively decrease during a sustained (120 s) MVC of the KE following exhaustive cycling in the heat, whereas the decrease in force production is significantly less pronounced and VA remains elevated after exercise in cool conditions. More recent studies have reported that VA and strength losses are equivalent during brief (5s) and sustained (20 s) contractions conducted after intense self-paced and maximal incremental exercise in hot and cool conditions. ${ }^{20} 21$ Part of the discrepancy may relate to the length of contraction. Indeed, after $30 \mathrm{~s}$ of maximal isometric effort, pain becomes increasingly severe, altering the perception of sensations originating from the active musculature, which leads to uncertainty as to the level of force being exerted. ${ }^{27}$ Moreover, a lack of motivation during protracted MVCs might reduce central activation to the appropriate motor neurons, resulting in the loss of tension. ${ }^{28}$ In the current study, the greater deficit in KE VA noted in the hot condition may have originated from a similar lack of motivation, decreasing the ability to voluntarily recruit (ie, brief MVC) and maintain (ie, sustained MVC) all available motor neurons, despite the feedback provided and the participants being constantly reminded to produce/sustain maximal torque. It may also have stemmed from a failure in descending drive (ie, insufficient motor unit firing rate) to compensate for a hyperthermia-induced increase in muscle relaxation rate. ${ }^{15} 17$

In contrast, the decrease in brief $(\sim 11 \%)$ and sustained $(\sim 14 \%)$ PF torque observed postmatch in the hot condition was associated mainly with a decline in twitch mechanical responses (table 2), as the $\sim 3 \%$ decrease in VA did not reach statistical significance (figure 2 and 4). Moreover, the decrement did not differ between hot and cool conditions. This is akin to soccer match data in which PF torque was reduced postmatch in hot and cool conditions, in association with a decrease in peak twitch torque $(\sim 10 \%)$ and a modest decrement in VA $(\sim 1.5 \%) .^{22}$ The difference in the extent of VA reductions between the KE and PF has previously been observed following ultra-marathon running in temperate conditions ${ }^{29} 30$ and incremental exercise to exhaustion in hot and cool conditions, ${ }^{21}$ suggesting that the reduction in central drive is not as severe in the $\mathrm{PF}$ as in the KE. This likely relates to muscle fibre composition, as the quadriceps have a higher percentage of type II fibres. The higher recruitment threshold of these fibres is suggested to result in a greater difficulty in fully activating this muscle group. ${ }^{3132}$ Of note, because of their role in producing powerful actions at high contraction velocities, ${ }^{33}$ fatigue or damage to type II muscle fibres (ie, fast-twitch fatigue-sensitive fibres) may result in reducing on-court movement efficiency and stroke proficiency. Hence, performance may become compromised during 
prolonged match-play tennis, especially under heat stress, owing to the progressive development of peripheral fatigue and a greater level of central fatigue in large muscle groups (eg, quadriceps).

\section{Recovery kinetics}

In temperate conditions, it has previously been shown that $\mathrm{KE}$ strength remains depressed $30 \mathrm{~min}$ in the recovery period following $3 \mathrm{~h}$ of match-play tennis. ${ }^{9}{ }^{10}$ It has also been shown that isometric bilateral leg-press force is reduced prior to the start of play on days two and three of a 3-day tournament, in parallel with increases in markers of muscle damage (ie, creatine kinase and muscle soreness). ${ }^{12}$ Data from soccer indicate that quadriceps muscle strength, along with muscle soreness, creatine kinase and muscle glycogen levels, also remain compromised $24 \mathrm{~h}$ after match completion. ${ }^{34} 35$ In contrast, PF torque production has been demonstrated to return to prematch level within $24 \mathrm{~h}$ of soccer play. $^{22}$ In the current study, voluntary torque production capacity, along with any postmatch adjustments in contractile function and central neural drive, was restored to prematch level within $24 \mathrm{~h}$ of playing in cool conditions for the KE and PF. Correspondingly, Kraemer et $a l^{36}$ postulated that a $24 \mathrm{~h}$ recovery period allowed for neuromuscular performance characteristics to fully recover following successive days of indoor match-play tennis competition. Interestingly, however, the authors commented that mental and physical perceptions of fatigue could still persist.

In the hot condition, KE torque during the brief and sustained MVCs remained depressed $24 \mathrm{~h}$ into recovery (figures 2 and 3). The decrement was associated with a significant reduction in VA during the sustained, but not the brief, contraction. This decrease in VA and the accompanied reduction in torque may relate to the persistent perception of psychological and physical fatigue, ${ }^{36} 37$ especially considering the return to baseline of contractile properties. Our findings, therefore, suggest that a certain level of mental fatigue may persist in the recovery period after play in the heat, whereby a sustained maximal isometric effort might be more difficult to reproduce with a large muscle mass high in fast-twitch fibres, such as the KE. ${ }^{37}$ Alternatively, it appears that $24 \mathrm{~h}$ is sufficient for the PF to recover from match-play tennis in hot and cool conditions and return to prematch strength capacity.

\section{CONCLUSIONS}

This study has shown that knee-extensor and plantar-flexor muscle strength is compromised following match-play tennis in hot and cool conditions due to significant levels of peripheral fatigue. In the knee-extensors, the loss of strength is exacerbated following play in the heat in association with larger reductions in central neural drive, which persists $24 \mathrm{~h}$ into recovery when performing sustained contraction. These alterations in neuromuscular system integrity have the potential to negatively influence the efficiency of on-court movements and stroke proficiency during match-play tennis, as well as in the recovery period. Match-related physical performance may, therefore, be compromised during prolonged matches and tournament play, especially under heat stress, owing to the progressive development of fatigue in large muscle groups, such as the quadriceps.

\section{PERSPECTIVES}

Given the specific role of the knee-extensors (ie, rapid on-court movements and explosive stroke production) and plantar-flexors (ie, positioning to the ball) in performing tennis movements and actions, fatigue in these muscle groups could impact play acutely in one-off matches and persistently with potential exacerbation across rounds of play, due to the cumulative effect. Future research should focus on enhancing our understanding of the mechanisms of strength loss, especially those linked to central activation failure, identifying potential training models or countermeasures to minimise neuromuscular perturbations during play, and on strategies to accelerate the recovery process in the lower limbs.

\section{What are the new findings?}

- Brief and sustained maximal voluntary strength is reduced in the lower limbs (ie, knee extensors (KE) and plantar flexors) immediately following match-play tennis in hot and cool conditions due to the development of peripheral fatigue.

- The reduction in KE strength is exacerbated after play in the heat due to a greater level of central fatigue.

- The loss of strength in the KE persists in the recovery period (24 h) after play in the heat, owing to a reduction in voluntary activation during sustained contractions.

- In the plantar flexors, the impairment in strength following match-play is similar between the hot and cool conditions, recovering within $24 \mathrm{~h}$, and appears mainly due to alterations in skeletal muscle mechanical responses.

Acknowledgements The authors thank all the participants for their participation in this study. The authors also thank the coaches, Tim Colijn and Samuel Rota, for conducting the practices during the days between matches.

Contributors JDP, OG and SR contributed to the development of the project, collection and analysis of data and have had intellectual input in drafting the manuscript and gave final approval for submitting the manuscript.

Funding This project was funded by the Aspire Zone Foundation Research Grant.

Ethics approval The study was approved by the Shafallah Medical Genetics Center Ethical Research Committee and conformed to the current Declaration of Helsinki guidelines

\section{Competing interests None.}

Provenance and peer review Not commissioned; externally peer reviewed.

Open Access This is an Open Access article distributed in accordance with the Creative Commons Attribution Non Commercial (CC BY-NC 3.0) license, which permits others to distribute, remix, adapt, build upon this work non-commercially, and license their derivative works on different terms, provided the original work is properly cited and the use is non-commercial. See: http://creativecommons.org/ licenses/by-nc/3.0/

\section{REFERENCES}

1 Fernandez J, Mendez-Villanueva A, Pluim BM. Intensity of tennis match play. Br J Sports Med 2006;40:387-91.

2 Ferrauti A, Bergeron MF, Pluim BM, et al. Physiological responses in tennis and running with similar oxygen uptake. Eur J Appl Physiol 2001;85:27-33.

3 Pluim B. Physiological demands of the game. In: Pluim B, ed. From breakpoint to advantage: a practical guide to optimal tennis health and performance. Vista, CA: USRSA, 2004:17-23.

4 Girard O, Millet GP. Neuromuscular fatigue in racquet sports. Neurol Clin 2008;26:181-94

5 Hornery DJ, Farrow D, Mujika I, et al. Fatigue in tennis: mechanisms of fatigue and effect on performance. Sports Med 2007:37:199-212.

6 Gandevia SC. Spinal and supraspinal factors in human muscle fatigue. Physio/ Rev 2001;81:1725-89.

7 Fabre JB, Martin V, Gondin J, et al. Effect of playing surface properties on neuromuscular fatigue in tennis. Med Sci Sports Exerc 2012:44:2182-9.

8 Girard O, Racinais S, Micallef JP, et al. Spinal modulations accompany peripheral fatigue during prolonged tennis playing. Scand J Med Sci Sports 2011;21:455-64

9 Girard O, Lattier G, Micallef JP, et al. Changes in exercise characteristics, maximal voluntary contraction, and explosive strength during prolonged tennis playing. $\mathrm{Br} \mathrm{J}$ Sports Med 2006:40:521-6. 
10 Girard O, Lattier G, Maffiuletti NA, et al. Neuromuscular fatigue during a prolonged intermittent exercise: application to tennis. J Electromyogr Kinesiol 2008;18:1038-46.

11 Edwards RG, Hill DK, Jones DA. Fatigue of long duration in human skeletal muscle after exercise. J Physiol 1977;272:769-78.

12 Ojala T, Häkkinen K. Effects of the tennis tournament on players' physical performance, hormonal responses, muscle damage and recovery. J Sport Sci Med 2013:12:240-8.

13 Périard JD, Caillaud C, Thompson MW. Central and peripheral fatigue during passive and exercise-induced hyperthermia. Med Sci Sports Exerc 2011:43:1657-65.

14 Morrison SA, Sleivert GG, Cheung SS. Passive hyperthermia reduces voluntary activation and isometric force production. Eur J Appl Physiol 2004;91:729-36.

15 Todd G, Butler JE, Taylor JL, et al. Hyperthermia: a failure of the motor cortex and the muscle. J Physiol 2005;563:621-31.

16 Thomas MM, Cheung SS, Elder GC, et al. Voluntary muscle activation is impaired by core temperature rather than local muscle temperature. J App/ Physiol 2006;100:1361-9.

17 Périard JD, Christian RJ, Knez WL, et al. Voluntary muscle and motor cortical activation during progressive exercise and passively induced hyperthermia. Exp Physiol 2014;99:136-48.

18 Racinais S, Gaoua N, Grantham J. Hyperthermia impairs short-term memory and peripheral motor drive transmission. J Physiol 2008;586:4751-62.

19 Nybo L, Nielsen B. Hyperthermia and central fatigue during prolonged exercise in humans. J Appl Physiol 2001;91:1055-60.

20 Périard JD, Cramer MN, Chapman PG, et al. Neuromuscular function following prolonged intense self-paced exercise in hot climatic conditions. Eur J Appl Physiol 2011;111:1561-9.

21 Racinais S, Girard 0 . Neuromuscular failure is unlikely to explain the early exercise cessation in hot ambient conditions. Psychophysiology 2012;49:853-65.

22 Nybo L, Girard O, Mohr M, et al. Markers of muscle damage and performance recovery following exercise in the heat. Med Sci Sports Exerc 2013;45:860-8.

23 Girard O, Bishop DJ, Racinais S. Neuromuscular adjustments of the quadriceps muscle after repeated cycling sprints. PLOS ONE 2013;8:e61793.
24 Merton PA. Voluntary strength and fatigue. J Physiol 1954;123:553-64.

25 Hill CA, Thompson MW, Ruell PA, et al. Sarcoplasmic reticulum function and muscle contractile character following fatiguing exercise in humans. J Physiol 2001;531:871-8

26 Westerblad H, Duty S, Allen DG. Intracellular calcium concentration during low-frequency fatigue in isolated fibres of mouse skeletal muscle. J App/ Physiol 1993;75:382-8

27 Bigland-Ritchie B, Jones DA, Hosking GP, et al. Central and peripheral fatigue in sustained maximum voluntary contractions of human quadriceps muscle. Clin Sci Mol Med 1978:54:609-14

28 Enoka RM, Stuart DG. Neurobiology of muscle fatigue. J Appl Physiol 1992; 72:1631-48

29 Martin V, Kerherve $H$, Messonnier LA, et al. Central and peripheral contributions to neuromuscular fatigue induced by a $24-\mathrm{h}$ treadmill run. J App/ Physiol 2010;108:1224-33.

30 Millet GY, Tomazin K, Vergès $S$, et al. Neuromuscular consequences of an extreme mountain ultra-marathon. PLOS ONE 2011;22:e17059.

31 Behm DG, Whittle J, Button D, et al. Intermuscle differences in activation. Muscle Nerve 2002;25:236-43.

32 Henneman E, Clamann HP, Gillies JD, et al. Rank order of motoneurons within a pool: law of combination. J Neurophysiol 1974;37:1338-49.

33 Beelen A, Sargeant AJ. Effect of fatigue on maximal power output at different contraction velocities in humans. J App/ Physiol 1991;71:2332-7.

34 Krustrup P, Ørtenblad N, Nielsen J, et al. Maximal voluntary contraction force, SR function and glycogen resynthesis during the first $72 \mathrm{~h}$ after a high-level competitive soccer game. Eur J Appl Physiol 2011;111:2987-95.

35 Rampinini E, Bosio A, Ferraresi I, et al. Match-related fatigue in soccer players. Med Sci Sports Exerc 2011:43:2161-70.

36 Kraemer WJ, Piorkowski PA, Bush JA, et al. The effects of NCAA division 1 intercollegiate competitive tennis match play on recovery of physical performance in women. J Strength Cond Res 2000;14:265-72.

37 Knicker AJ, Renshaw I, Oldman ARH, et al. Interactive processes link the multiple symptoms of fatigue in sport competition. Sports Med 2011;41:307-28. 